\title{
A thermographic study for the assessment of historic structures
}

\author{
by N.P. Avdelidis ${ }^{*}$, A. Moropoulou** and E.T. Delegou** \\ * IRT \& Materials Consultancy, Agia Triada Ano Volos, Volos 38500, Greece. \\ ** Materials Science \& Engineering Section, National Technical University of Athens, \\ Athens 15780, Greece.
}

\begin{abstract}
Although, the efficiency of thermography as a non-destructive technique in the literature it is well documented, in the investigation of historic structures, where a restoration or conservation treatment can cause irreversible damage to the structure, it is considered to be of most importance. In the present work, both thermographic approaches, passive and active, were used on two different applications, for the investigation of historic materials and structures. The technique was applied on restoration and traditional-historic materials and structures for the evaluation of conservation interventions (materials and techniques) concerning cleaning of architectural surfaces, as well as for the disclosure of tesserae on a plastered mosaic surface.
\end{abstract}

\section{Introduction}

Infrared thermography has been applied for more than 25 years in buildings and historic structures monitoring. Although this technique has the potential to deliver first class results as far as detection and measurement of damage in a material is concerned, the interpretation of a thermographic survey can be fairly complex. During an infrared thermographic investigation of a building material or a structure, there are various physical properties that need to be considered. Those are thermal properties (i.e. conductivity, diffusivity, effusivity and specific heat), spectral properties (i.e. absorption, emissivity, reflection and transmission) and other properties characteristics (i.e. porosity, volumetric mass and physiological water content).

All of the above mentioned characteristics are significant in order to realise the outcome of a thermographic survey. For instance, a material with voids or pores, decreases its thermal conductivity and density, its thermal diffusivity is altered and so the conduction of heat transfer within the material is affected. This can be realised when considering equation 1 :

$$
a=\frac{k}{\rho C_{p}}
$$

where:

$\mathrm{a}$ is the thermal diffusivity $\left(\mathrm{m}^{2} \mathrm{~s}^{-1}\right), \mathrm{k}$ is the thermal conductivity $\left(\mathrm{Wm}^{-1} \mathrm{~K}^{-1}\right), \rho$ is the density $\left(\mathrm{kgm}^{-3}\right)$ and $\mathrm{Cp}$ is the specific heat capacity $\left(\mathrm{Jkg}^{-1} \mathrm{~K}^{-1}\right)$.

Another example is when the investigated material presents moisture. In such cases, the optical properties are altered, the density, specific heat capacity and thermal conductivity are also affected and so any temperature changes are much slower in a moist area. In other words, the energy required to raise the temperature 
of a moist area would be much greater than an area that is unaffected by water. This can be understood from the following equation:

$$
Q=m C p \Delta T
$$

where:

$Q$ is the absorbed energy (Joules), $m$ is the mass $(\mathrm{Kg}), \mathrm{Cp}$ is the specific heat capacity $\left(\mathrm{Jkg}^{-1} \mathrm{~K}^{-1}\right)$ and $\Delta \mathrm{T}$ is the change in temperature $(\mathrm{K})$.

Another consideration when a thermographic survey is to be completed is the thermal effusivity of the materials to be tested. Materials with low effusivity values will increase rapidly temperature, since:

$$
T_{(t)}=\frac{Q}{e \sqrt{\pi t}}
$$

where:

$\mathrm{Q}$ is the input energy (Joules) and $\mathrm{e}$ is the thermal effusivity $\left(\mathrm{Ws}^{1 / 2} \mathrm{~m}^{-2} \mathrm{~K}^{-1}\right)$, which can be calculated by:

$$
e=\sqrt{k \rho C_{p}}
$$

In order to investigate a material or a structure by thermal means, there are two approaches that can be used; passive and active. In the passive approach, commonly used for civil engineering structures inspection, the material under investigation is frequently inspected in terms of qualitative means. On the contrary, since in the active approach a thermal excitation source is used, it is also possible to attain quantitative results. This is can be achieved because the heating or cooling features of the excitation source are specified in time and amplitude. However, when a material is heated, the thermal waves penetrate the material's surface. These waves are generally of various amplitudes and frequencies and are launched into the specimen, in a transient mode. The thermal diffusion length is then expressed by:

$$
\mu=\sqrt{\frac{\alpha}{\pi f}}
$$

where:

$\mathrm{f}$ is the frequency $(\mathrm{Hz})$.

So, since the thermal diffusion length is inversely proportional to the frequency, then at high frequencies there will be a near to surface analysis, whilst at low frequencies the thermal waves will propagate deeper, but also slowly, since the speed is given by:

$$
V=\sqrt{4 \pi f \alpha}
$$

\section{Thermographic investigation}

Thermography was applied on various materials and structures in order to assess, physico-chemically, conservation treatments such as stone cleaning, as well as to disclose the original mosaic tesserae beneath a plastered mosaic surface. In the first case the passive approach was used, whilst in the plastered mosaic examination, active thermography, (using a heating source), was employed. The 
thermographic system used consists of an IR detector (TVS 2000 Mk II LW) and a processor (AVIO Thermal Video System). The IR detector is constructed of mercurycadmium-telluride that gives a spectral response between 8 and $12 \mu \mathrm{m}$, with a scan speed of 15 frames per second.

\section{Surface cleaning assessment}

The cleaning of stone and architectural surfaces results in aesthetic and physicochemical alterations to the applied surface. According to British Standard 8221-1:2000, buildings are cleaned either to enhance their appearance or to assist maintenance and/or conservation.

There are various cleaning techniques, ranging from those that are intended for use on large facades to those that are intended for meticulous use on finely carved and delicate sculpture. Their efficiency is commonly assessed subjectively. Infrared thermography can be employed in order to assess any surface alterations (i.e. roughness) after a cleaning application, as well as to investigate any moisture deposits in the examined surfaces [1].

As an example of this type of work, an infrared thermographic investigation took place in two of the most representative buildings for neoclassical architecture in Athens, the Athens Academy and the National Library of Greece. Two surfaces consisting of white Pentelic marble, a metamorphic limestone consisting almost exclusively of calcite grains and quarried from mountain Penteli in Athens, were investigated. Photos of the surfaces are shown in figures $1 \mathrm{a}$ and $1 \mathrm{~b}$.

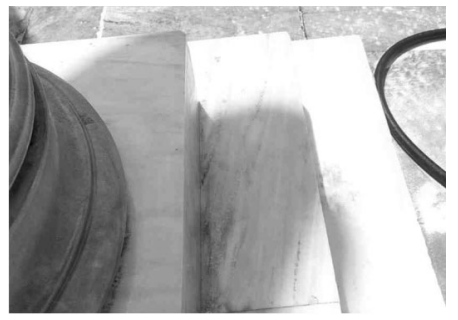

(a) Athens Academy

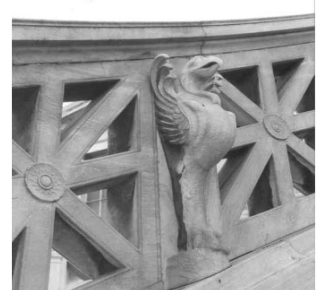

(b) National Library

Fig. 1. Photos of investigated marble surfaces

A wet micro blasting cleaning system was utilised as a pilot cleaning intervention on the investigated surfaces. Spherical particles of calcium carbonate, with diameter lower than $80 \mu \mathrm{m}$, were blown with several function pressures (ranging from 0.5 to $1 \mathrm{~atm}$ ) on the examined surfaces.

The surfaces were characterised by different decay patterns, i.e. black-grey crusts, organic depositions, washed out surfaces. Thus, the efficiency of the cleaning application in such circumstances was also assessed. The proportion of water and spherical particles of calcium carbonate was 2:1, while the nozzle's internal diameter was $2 \mathrm{~cm}$. The suspension's percussion angle, function pressure values and nozzle's distance from the treated surfaces were also reported [2]. The measurements in the field, as well as the cleaning interventions were applied during mild environmental conditions.

The main advantage of the applied cleaning method is that it appears to have tolerable abrasion levels due to the low values of Mohs hardness (approximately 3) of the calcium carbonate spherical particles, in comparison to all other wet particleblasting methods that generally use alumina particles (Mohs hardness of 8 to 9). 


\section{http://dx.doi.org/10.21611/qirt.2004.054}

Before and after the cleaning, infrared thermography (Avio TVS 2000 Mk II LW, 8-12 $\mu \mathrm{m}$ ) was performed in situ on the examined surfaces. In the Athens Academy case (figure 2), since great amounts of water were used during the cleaning application (wet micro blasting cleaning method), significant quantities of moisture within the examined surfaces were detected; because of the difference in specific heat capacity, density and thermal conductivity for both water and marble. Since the specific heat of water is approximately five times greater than marble, this would mean that any changes in temperature would be much slower in an area contaminated by water.

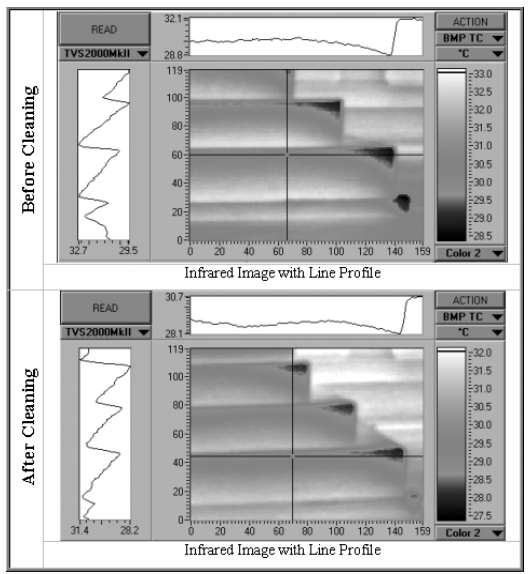

Fig. 2. Thermography results after investigating the Athens Academy surface

In the case of the National Library of Greece historic building (figure 3), the temperature of the cleaned surface was increased. It is most likely that an increase of the surface roughness influenced the propagation of radiant heat from the sun on the surface, altering the emittance of the surface. So, a contrast between smooth and rough surfaces was observed.

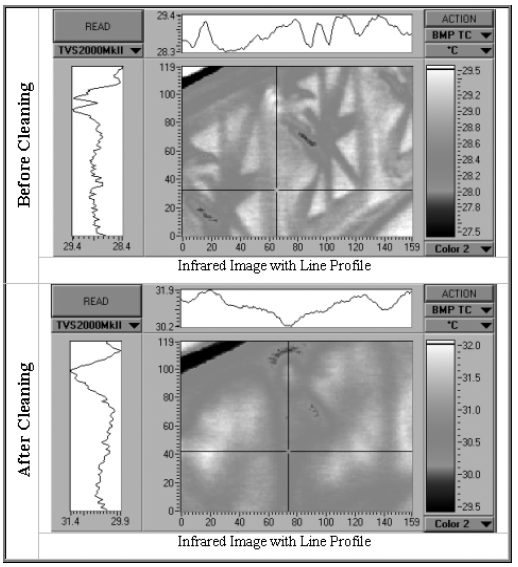

Fig. 3. Thermography results after investigating the surface at the National Library of Greece 
Comparing the thermal images and in particular the plotted line profiles obtained before the cleaning intervention with those after cleaning, a considerable reduction of the temperature variance was noticed. This is due to the fact that the surfaces present greater homogeneity after the cleaning treatments, since the removal of most types of decay patterns was successful.

\section{Evaluation of a plastered mosaic surface}

There have been various works where the employment of non-destructive testing techniques provided substantial information attainment concerning materials examination. Principally, infrared thermography has been utilised for applications such as moisture assessment in buildings [3] and detection of sub-surfaces [4].

Hagia Sophia in Istanbul is one of the unique monuments where assorted mosaic categories of invaluableness are offered. However, interventions in the past have triggered aesthetic problems leading to some cases to severe damage of the mosaic. Such example is the intervention occurred in the early 1900's, under the General Directorate of Pious Foundations, mostly known as the "Vakif intervention". The plaster of this intervention was covered with an opaque yellow pigment in a glue medium. Stencil ornaments in pattern were applied on this yellow ground [5].

In a previous research work by two of the authors and others [6], various types of mosaics were investigated in the laboratory using infrared thermography, in order to reveal the mosaic - tesserae beneath the plastered surfaces. Due to the different thermal diffusion that each layer renders, infrared thermography was able to detect the different sub-surfaces on the plastered mosaics, presented with temperature variations on the surfaces. This was accomplished after employing the active thermographic approach, using an external optical heat source in order to uniformly heat the inspected panels.

In this work, a plastered mosaic surface (characteristic Vakif intervention surface) in the Hagia Sophia dome was investigated. The surface was situated between the 19th and the 20th rib at the northwest part of the dome, 16 metres from the centre of the dome. Figure 4 shows the examined surface whilst a thermal image obtained from the investigation of this surface is shown in figure 5 .

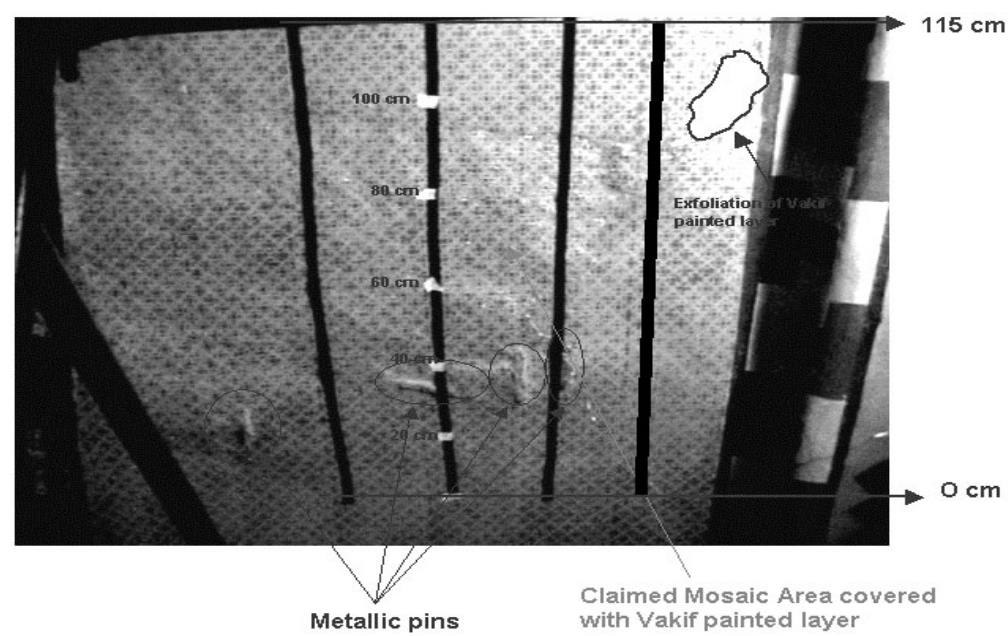

Fig. 4. Photograph of investigated surface in the Hagia Sophia dome 


\section{http://dx.doi.org/10.21611/qirt.2004.054}

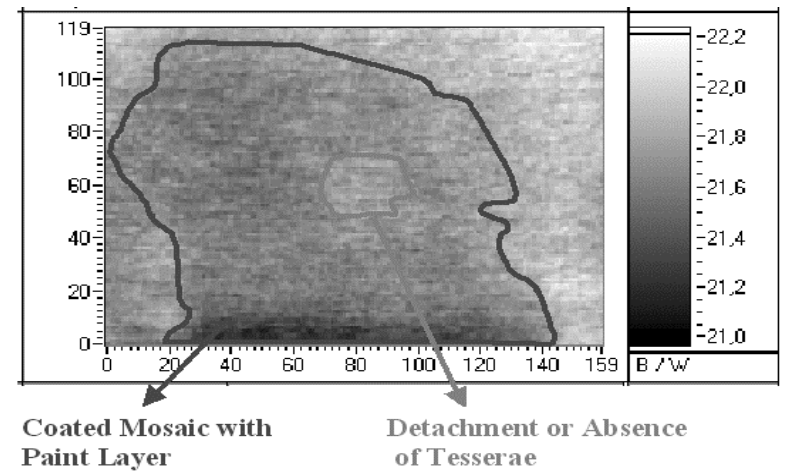

Fig. 5. Thermal image of the investigated plastered mosaic surface

The surface was heated by powerful optical lamps and during the cooling down process the presented thermal image was obtained. The image illustrates the existence of the coated mosaic, as well as the detached area within it. Whilst, darker grey areas - lower temperature values - represent the mosaic, the detached areas, behaving similar to a sub-surface defect, hold remarkably higher temperature values, due to the different thermal diffusion rate (thermal contrast).

\section{Conclusions}

The main aim of this work was to present the efficiency of thermography as a non-destructive testing and evaluation technique in the investigation of historic structures. It is realised that the technique offers significant information for the assessment of materials and techniques presented in this work. Particularly, it can be used effectively in the assessment of conservation materials and techniques on the subject of surface cleaning, as well as to disclose any substrate features, such as tesserae on a plastered mosaic surface.

\section{REFERENCES}

[1] Avdelidis, N.P. and Moropoulou, A. Review Paper: Applications of infrared thermography for the investigation of historic structures, Journal of Cultural Heritage, 5, 119-127, (2004).

[2] Moropoulou, A., Delegou, E.T., Avdelidis, N.P. and Koui, M. Assessment of cleaning conservation interventions on architectural surfaces using an integrated methodology, Materials Issues in Art and Archaeology VI, Editors Vandiver, P., Goodway, M., Druzik, J.R. and Mass, J.L. 69-76, Pittsburgh, (2002).

[3] Titman, D.J. Applications of thermography in non-destructive testing of structures, Journal of NDT \& E International, 34, 149-154, (2001).

[4] Avdelidis, N.P. and Almond, D.P. Transient thermography as a through skin imaging technique for aircraft assembly: modelling \& experimental results, Journal of Infrared Physics \& Technology, 45, 103-114, (2004).

[5] Ozil, K.R. The conservation of the dome mosaics of Hagia Sophia, Light on Top of the Black Hill - Studies presented to Halet Cambel, 543-552, (1998).

[6] Moropoulou, A., Avdelidis, N.P., Aggelakopoulou, E., Griniezakis, S., Koui, M., Aggelopoulos, A., Karmis, P. and Uzunoglou, N.K. Examination of plastered mosaic surfaces using NDT techniques, Journal of Insight, 43, 241-243, (2001). 